\title{
INFLUENCE OF BUDDHIST LITERATURE IN THE WORKS OF RABINDRANATH
}

\author{
Dr. Shekhar Purkayastha
}

\begin{abstract}
Rabindranath was greatly influenced by Buddhist ideals. According to him Buddhism was one of the greatest religions preaching universal love. This paper is an analytical study of some of Rabindranath's poems namely 'Pujārini', 'Shrestha Bikshā', 'Parishodh' and dramas namely 'Achalāyatan', Mālini', 'Chandālikā' (dance drama) which are adaption of the stories from Avadānaśataka, a book on Buddhist fables composed by unknown poet. An attempt has been made to show how the poet's deep respect for the teachings of Buddhism has been reflected in his literary works based on the stories narrated in Avadānaśataka.
\end{abstract}

যে বিশ্বভ্রাতৃত্ব বা বিশ্বপ্রেম রবীন্দ্র জীবন দর্শনের মূল অবলম্বন বৌদ্ধধর্নে সেই মৈত্রী বা প্রেমই সাধনার চরম উৎকর্য রূপে প্রতিষ্ঠিত হয়েছে। বুদ্ধদেবের নৈত্রীসাধনার মধ্যে বিশ্বমানবের যে মিলনসূত্র অভিব্যক্ত কবিকে তা বিশেষভাবে আকর্ষণ করেছে। বুদ্ধেব জীবন ও বাণী কবির অন্তরে গভীর প্রভাব বিস্তার করেছিল, তাই তিনি বিশ্বাস করতেন বুদ্ধ বিশ্বের সর্ব(্রেষ্ঠ মানব। মানব জীবনের পরম প্রাপনীয় বস্তু কোনো রাজসিংহাসন নয়, রাজঐশ্বর্যও নয়, সর্বমানবের প্রতি পরম প্রেমই হচেছ মানব জীবনের অন্বিষ্ট বস্তু। অন্যের প্রতি সেই প্রেমকে বিলিয়ে দেওয়াতেই মানুযের জীবন ধন্য হয়ে যায়। বুদ্ধের মট্যে সেই পরম প্রেনেরই পূর্ণ প্রকাশ কবি দেখতে পেয়েছিলেন। তাই বুদ্ধদেব সম্পর্কে তিনি লিখেছেন“ভগবান বুদ্ধ তপস্যার আসন থেকে উঠে আপনাকে প্রকাশিত করলেন। তাঁর সেই প্রকাশের আলোকে সত্য দীপ্তিতে প্রকাশ হল ভারতবর্ষের। মানব ইতিহালে তাঁর চিরন্তন আবির্ভাব ভারতবর্ষের ভৌগোলিক সীমা অতির্রমম করে ব্যাপ্ত হল দেশে দেশান্তরে। ভারতবর্ষ তীর্থ হয়ে উঠল, অর্থাৎ স্বীকৃত হল সকল দেশের দ্বারা, কেননা বুদ্ধের বাণীতে 
ভারতবর্ষ সেদিন স্বীকার করেছে সকল মানুষকে।"’ - সুতরাং কবিকে বুদ্ধদেব কত গভীরভাবে প্রভাবিত করেছিলেন তা আমরা সহজেই অনুমান করতে পারি। সেজন্যেই তাঁর বহু সাহিত্য সৃষ্টিতে বৌদ্ধ সাহিত্যের ও বৌদ্ধ দর্শনের প্রভাব আমরা দেখতে পাই।

রাজেন্দলাল মিত্রের অনুরোধে হরপ্রসাদ শাস্ত্রী সমগ্র ‘মহাবস্তু অবদান’ সংক্ষিপ্ত করে দিয়েছিলেন, তার উপর নির্ভর করে রাজেন্দলাল মিত্র তাঁর “The Sanskrit Buddhist Literature of Nepal (Cal, 1882)” প্রকাশ করেন। রবীন্দ্রনাথ সেই সংক্ষিপুসার বৌদ্ধ কাহিনিগুলি নিবিষ্টভাবে পড়াশোনা করেছিলেন। তার ফলস্বরূপ তিনি বৌদ্ধকাহিনি অবলম্বনে কিছু নাটক এবং কবিতা রচনা করেন। এই কবিতা এবং নাটক গুলিতে বৌদ্ধযুগের ভারতবর্ষের এক জীবন্তরূপকে আমরা পেয়ে থাকি। রবীন্দ্র সৃষ্টি নিজচ নৌলিক দর্শনের আলোকে মূর্ত হয়ে উঠলেও এই নাটক ও কবিতাগুলির মূল সূত্র নিহিত রয়েছে ঐ অবদান সাহিত্যে। রবীন্দ্রনাথ সৃষ্ট ঐ কবিতা ও নাটকগুলির আলোচনা করলে তা আমরা খুব সহজেই বুঝতে পারব।

রবীন্দ্রনাথের 'রাজা’ নাটকের আখ্যানভাগ মহাবস্তু অবদানের অন্তর্ভুক্ত ‘কুশজাতক’ এর কাহিনি থেকে গৃহীত। রাজেন্দ্রলাল মিত্রের উন্নিখিত গ্রন্থে 'The Story of Kusa' রবীন্দ্রনাথ পড়েছিলেন। यদিও মূল মহাবস্তু অবদানের সঙ্গে তাঁর পরিচয় ছিল কিনা তা জানা যায়না। রবীন্দ্রনাথ যে অংশটি থেথেক ‘রাজা' নাটকের বিষয়বস্তু গ্রহণ করেছেন তার সংক্ষিপ্তসার এরকম-

বারাণসীর মহারাজা ইক্ষাকুর প্রধানা মহিষী অলিন্দার সন্তান কুশ বা শুদ্ধকুশ। কুশ অতীব সুদর্শন কিন্তু অশেষ গুণী, সর্ববিদ্যায় পারদর্শী। যৌবনে পদার্পণ করলে রাণী অলিন্দা তাঁর বিয়ের ব্যবস্থা করেন। অলিন্দার কৌশলে কাহ্কুক্জরাজ মহেন্দ্রকের কন্যা সুন্দরী সুদর্শনার সঙ্গে কুশের বিবাহ হয়। কুলপ্রথার অজুহাতে কৌশল করে অলিন্দা এক অন্ধকার গৃহ নির্মাণ করান, সেখানে কুশের সঙ্গে সুদর্শনার মিলন হত। বাইরের আলোতে কুশ ও সুদর্শনা একে অপরকে দেখতে পেতনা- "She therefore prepared rooms underground where under the plea of family custom, she placed the young couple. No light was admitted into the rooms. The couple enjoyed their honeymoon in the dark."

রাণী সুদর্শনা অধীর হয়ে রাজাকে দিনের আলোয় দেখতে চান। পাছে সুদর্শনা কুৎসিৎ কুশকে দেখে ফেলেন এই ভয়ে অলিন্দা কুশের বৈমাত্রেয় ভাই কুশদ্রুম্কে সিংহাসনে বসিয়ে জানালা দিয়ে সুদর্শনাকে দেখিয়ে বলেন- ‘ঐ কুশ’। নকল কুশকে দেখে রানী খুশী হন, কিন্তু পাশেই রাজছত্রধারী কদাকার কুশ্রী সম্পন্ন আসল কুশকে দেখে বিরক্ত হন। পরে সত্য প্রকাশিত হলে কুলের ভয়ংকর চেহারা দেখে অত্যন্ত ঘৃণায় ও আতঙ্কে রাজধানী ছেড়ে কাহ্কুকে বাপের বাড়ি চলে যান। এদিকে সুদর্শনার অদর্শন কুশের মনে তীব্র বিরহের সঞ্চার করে। তিনি তাঁর ভায়ের মতো এক ব্যক্তিকে রাজ সিংহাসনে বসিয়ে এক বীনা হাতে নিয়ে সুদর্শনার উদ্দেশ্যে কাহ্নকুক্ডে চলে যান। সেখানে পৌঁছে শিল্প ও কলা বিদ্যায় অশেষ 
গুণপনার পরিচয় দিয়ে কুশ রাজবাড়ির পাক শালায় কাজ পেলেন। সেখানে ছদ্মবেশে থেকে সুদর্শনার মন জয় করতে তিনি ব্যর্থ হন।

ইতিমধ্যে সুদর্শনার স্বামীগৃহ ত্যাগের সংবাদ কাহুকুক্ডের পার্শ্ববর্তী রাজ্যের সনাতন রাজা সুদর্শনার পাণিপ্রার্থী কাহৃকুক্জরাজকে চিঠি লেখেন। তাঁরা প্রত্যেকেই সুদর্শনাকে বিয়ে করতে চান এবং তাযদি নাহয় তাহনে রাজ্য আব্রম্মণ করার ভয় দেখান। বাজা ভীযণ সংকটে পড়ে সুদর্শনাকে তিরস্কার করেন এবং তাকে সাতটুকরো করে কেটে সাত জনকে উপহার দেবেন স্থির করেন। সুদর্শনা ও রানী ভয়ে কান্নাকাটি করতে শুরু করেন। এমন সময়ে রাজার কুশের কথা স্মরণ হয়। সুদর্শনা তখন রাজবাড়িতে কুশের উপস্থিতির কথা পিতার কাছে প্রকাশ করেন। রাজা কুশকে পরম সম্মানে বিভূষিত করে আসন্ন বিপদ থেকে তাঁকে রক্ষা করবার জন্য অনুরোধ করেন। কুশ হস্তিপৃষ্ঠে আরোহণ করে যুদ্ধযাত্রা করেন এবং অসীম বীরত্ব প্রদর্শন পূর্বক সাতজন রাজাকেই বন্দী করে নিয়ে আসেন। পরিশেযে কুলের অনুরোধে কাহ্কুক্জরাজের অপর সাতকন্যাকে বন্দী সাতজন রাজার হাতে সমর্পণ করা হয়। সুদর্শনা তখন তাঁর ভুল বুঝতে পারেন। স্বামীর শ্রের্যবীর্যে মুগ্ধ হয়ে স্বামীর সঙ্গে শ্বশুরালয়ে যার্রা করেন। যাওয়ার পথে একটি ছোট্ট নদীর স্বচছ জনে নিজের বিকৃত দর্শন চেহারার প্রতিফলন দেখে কুশ মর্মাহত হন এবং জলে ডুবে মরতে ইচ্ছা করেন। ঠিক সেই সময়ে দেবরাজ ইন্দ্র সেখানে আসেন এবং তাঁকে ‘জ্যোতিরম’ নানে দুর্লভ একছড়া মণিমালা উপহার দেন। ইন্দ্র বলেন যখন খুশি কুশ এটি পড়লে তিনি তৎক্ষণাৎ পৃথিবীর সবচেয়ে সুন্দর পুরুষ হবেন এবং খুনে ফেলনে পূর্বরূপ ফিরে আসবে। কুশ সেই মনিমালা পরিধান করেন এবং সুদর্শনা তার স্বামীর সুন্দর রূপ মূর্তি এবং স্বর্গীয় সৌন্দর্য দেখে আনন্দে উৎফুল্লিত হয়ে ওঠেন। এখানে বুদ্ধ ছিলেন সেই কুশ, কাহ্কুক্ডরাজ মহেন্দ্রক ছিলেন মহানাম, অলিন্দা মায়া, সুদর্শনা যশোধরা এবং সেই বিদ্রোহী সাতরাজা ছিলেন মার এবং তার সঙ্গী।

এই কাহিনির উপব ভিত্তি করে ‘রাজা’ নাটকটি রচিত। রূংপক- সাংকেতিক ভাযা ব্যবহারের কৌশলে এক গভীর আধ্যাত্মিক তত্ত্বের উদ্ধোধনে ‘রাজা’ নাটক রবীন্দ্রনাথের এক স্বতন্ত্র সৃষ্টি বলা যেতে পারে। সমালোচক রাধাগোবিন্দ বসাক বলেছেন- "It must be acknowledged that Tagore's keeping infact the theme of the dark chamber in which the hero and the heroine lined and amused themselves without seeing each other, created two unique and brilliant pieces of drama in which he introduced many innovations and divergences from the original Mahavastu story for his own dramatic purpose."

রবীন্দ্রনাথ চঁঁর সৃষ্ট এই নাটকে যদিও কলা-কৌশল ও তত্ত্ব প্রয়োগের মাধ্যনে কাহিনির এক স্বতন্ত্র শিল্পমূল্য দান করেছেন, কিন্তু নাটকের উৎস সন্ধানে বৌদ্ধসাহিত্যেরই আশ্রয় গ্রহণ করেছিলেন। স্বাতন্ত্রের দিক্ থেকে আলোচনাকে আমরা অগ্রসর করে বিস্তৃত 
করবনা কারণ রবীন্দ্রনাথের কিছু সৃষ্টির উৎস বা অবলম্বন যে ব্বৌদ্ধসাহিত্যের দ্বারা বিশেষভাবে প্রভাবিত সেই দিকটিই আমাদের আলোচ্য বিষয়।

‘অচলায়তন' নাটকটিরও উৎসমূলে রয়েছে ‘দিব্যাবদানমালা’র অর্ত্তগত পঞ্চকের কাহিনি।" কাহিনিটি এরকম- এক ব্রাহ্মণের অনেকগুলি সন্তান একের পর এক জন্ম দিয়ে মারা যায়। পরে একটি সন্তান শ্রমণ-্রাদ্মণদের আশীর্বাদে বেঁচে যায়। তার নাম হল পঞ্চক। পরে আরও একটি সন্তান বাঁচে, তার নাম মহাপঞ্চক। বড়ো হরয়ে পঞ্চক ও মহাপঞ্চক উভয়েই সন্য্যাস গ্রহণ করে। কালত্রুন্মে মহাপঞ্চক একজন মহাজ্ঞানী হয়ে অর্হত্ব অর্জন করে, আর পঞ্চক মহামুর্থই থেকে যায়। মহাপঞ্চক সেজন্য বিহার থেকে তাকে তাড়িয়ে দেয়, বিতাড়িত পঞ্চক দুঃখে রাস্তার পাশে বসে কাঁদতে থাকে। ভগবান বুদ্ধ তাকে এরকম অবস্থায় দেখতে পেয়ে বিহারে নিয়ে আসেন। একজন ভিক্ষুর উপর পঞ্চকের ভার অর্পিত হয় এবং অল্পকালের মধ্যেই সে অর্হত্ব লাভ করে। পূর্বজন্মের সুকৃতির ফনেই নাকি ইহজন্মে পঞ্চকের পক্ষে অর্হত্ব লাভ সম্ভব হয়েছিল। এরপর অবদানে পূর্বজন্মের কাহিনি বিবৃত হয়েছে, এখানে তার উল্লেখ নিষ্প্রয়োজন। এই দিব্যাবদান কাহিনির ক্ষীণসূত্র অবলম্বন করে রবীন্দ্রনাথের ‘অচলায়তন’ নাটক রচিত হয়। যদিও চরিত্রের নামগুলি ছাড়া অচলায়তনের বক্তব্যের সঙ্গে পঞ্চকের কাহিনির কোনো যোগ নেই, উৎসের দিক্ থেকে দেখতে গেলে নাটকের চরিত্রগুনো অবদান সাহিত্য থেকেই গ্রহণ করা হয়েছে। কাহিনির দিক্ থেকে মিল না থাকলেও নাটকের মূল বিষয়বস্তুর সঙ্গে বৌদ্ধসাহিত্যের এই অংশটুকুর সাদৃশ্য খুঁজে পাওয়া যায়। রবীন্দ্রনাথ নাটকে অন্ধসংক্কার ও জরাজীর্ণ আচার সর্বস্ব ধর্মকে বিদ্রুপ করেছেন এবং সংস্কারমুক্ত মানবধর্মুর জয়গান গেয়েছেন। পঞ্চকের কাহিনিতেও মহাপঞ্চক জীর্ণ স্থিতিশীল, অন্ধ সামাজিক আচার নিষ্ঠার এবং পঞ্চক মুক্তি পিপাসু মানবাত্মার প্রতীক, কাজেই ‘অচলায়তন’ নাটকের উৎস হিলেবে বৌদ্ধসাহিত্যের এই অংশটুকুকে কিছুতেই অস্বীকার করা যায় না।

রবীন্দ্রনাথের 'মালিনী’ নাট্যকাব্যটির উৎস হচ্ছে ‘মহাবস্তু অবদান’ এব মালিনী উপাখ্যান ('মালিন্যা বস্তু' -The Story of Malini)। 'মহাবস্তু অবদানে'র মালিনী উপাখ্যানটি খুব বিস্তৃত। রাজেন্দ্রলাল মিত্রের ‘সংক্ষিপু অনুবাদ’ থেকেই রবীন্দ্রনাথ তা গ্রহুণ করেছেন বলা যায়। মূলকাহিনির যেটুকু বিষয়ের সঙ্গে ‘মালিনী’ নাটিকার মোটামোটি যোগ আছে তার আখ্যান বস্তুটি মোটামোটি এরকম - বারাণসীর মহারাজা কৃকির রুচিরা কন্যা মালিনী ব্রাম্মণদের পরিচর্যা করতেন। একসময়ে তাঁদের অশিষ্ট আচরতে অসন্তষ্ট হয়ে তিনি তাঁদের প্রতি অশ্রদ্ধা প্রদর্শন করেন এবং ভিক্ষু ভগবান কাশ্যপের প্রতি শ্রদ্ধাপরায়ণা হন। একদিন মালিনী সশ্রাবক সংঘ কাশ্যপের বন্দনা জানিয়ে রাজ অন্তঃপুরে অন্নগ্রহণের জন্য তাঁকে নিমন্ত্রণ করেন। ভগবান কাশ্যপ বিনয় শিক্ষার জন্য তাঁর প্রতি অনুকস্পা দেখিয়ে সেই নিমন্ত্রণ গ্রহণ করেন। ফলে সেই রাজ্যের সহস্র সহস্র ব্রান্মণ ত্রুন্দ্ধ হয়ে চঞ্রান্ত করেন এবং মালিনীকে মেরে ফেলতে চান। তাঁরা মালিনীকে পরিত্যাগ করে তাঁদের হাতে সমর্পণ করবার জন্য রাজার উপর চাপ সৃষ্টি করতে থাকেন। রাজা ভয়ে রাজ্য রক্ষার স্বার্থে 
রাজকন্যাকে বিক্ষুদ্ধ ব্রাহ্মণদের হাতে তুলে দেন। ব্রাহ্মণগণ মালিনীকে হত্যা করবার সিদ্ধান্ত করলে মালিনী দান-পুণ্য কাজের নিমিত্ত মাত্র একসপ্তাহের জন্য জীবন ভিক্ষা করেন। ব্রাম্মণেরা তাঁর অনুরোধ রক্ষা করেন। তখন মালিনী সেই সাতদিন ধরে আবার শ্রাবক সংঘসহ ভগবান কাশ্যপের সেবা ও দানরূপ পুণ্যকর্ম করতে থাকেন। সেই সাতদিনে ভগবান কাশ্যপ মহারাজা কৃকি, রাজ অমাত্য, প্রধান সেনাপতি, অন্তঃপুরজন এবং পৌরজনদের বিনয়ধর্মে দীক্ষিত করেন। আর্যধর্মে বীনিত তাঁদের মনে তখন এইভাব জাগে যে মালিনী তাঁদের কল্যাণকামী বন্ধু, তাঁকে পেয়েই তাঁরা সর্বধর্মে বিশুদ্ধ ধর্মচক্ষু লাভ করেছেন। তারপর তাঁরা নিজের জীবন দিয়েও মালিনীকে রক্ষা করবার শপথ গ্রহণ করেন। এদিকে বিক্ষুদ্ধ ব্রাহ্মণগণ সমবেত হয়ে হত্যার জন্য মালিনীকে অপহরণ করতে চান। রাজসেনা বাসস্থানের দিকে ধাবিত হলে তাঁরা ভয়ে পালিয়ে যান। তখন তাঁদের কোপ গিয়ে পড়ে ভগবান কাশ্যপ ও ভিক্ষুদের উপর। শ্রাবকসংঘ সহ কাশ্যপকে হত্যার জন্য তাঁরা বারে বারে বহু কপট বেশধারী ব্রাহ্মণদের প্রেরণ করেন। ভগবান কাশ্যপ তাঁর অলৌকিক ক্ষমতা বলে তাদের সকলকেই আর্য ধর্ন্ম দীক্ষিত করেন। তারপর কাশ্যপ উপদিষ্ট সেই ব্রাম্মণেরা ভগবানের মহিমা গান করতে থাকেন। তখন যে সকল ব্রান্মণ মিথ্যায় প্রতিষ্ঠিত ছিলেন তাঁরা দুর্বুদ্ধি কবলিত হয়ে সকলে মিলে দণ্ড ও লগুড় নিয়ে ভগবান কাশ্যপের দিকে ধাবিত হলেন। ভগবান পৃথিবীদেবতাকে আহ্বান করলেন, পৃথিবীদেবতা তাল বৃক্ষ দিয়ে সেই দুষ্ট ব্রাম্মণদের বেদম প্রহার করতে লাগলেন। তখন ভীত হুয়ে ব্রাদ্মণেরা পলায়ণ করেন।

রবীন্দ্রনাতের 'মালিনীতত কাহিনি উপস্থাপনে, রচনাচাতুর্যে, শিল্পকৌশলে অবদান কাহিনির সঙ্গে প্রচুর পার্থক্য থাকলেও মূলভাবের দিক্ থেকে দেখা যায় ধর্মের যথার্থ স্বরূপ যেমন মূল কাহিনিত সংস্থাপিত হয়েছে, সংকীর্ণতা, হিংসা-দ্বেষ যেমন সেখানে স্থান পায়নি ঠিক সেরূপই 'মালিনী' নাটিকায়ও বিশ্বজনীন মানবধর্মেরই জয় ঘোষিত হয়েছে। বুদ্ধের প্রেম-নৈত্রী করুণার সম্মিলিত প্রকাশ যে সত্য ধর্মের মধ্যে 'মালিনী’ নাটিকার সুপ্রিয় চরিত্রের ভায্যে তাই সুন্দরভাবে প্রকাশিত হয়েছে-

"যেথা দয়া সেথা ধর্ম, যেথা প্রেমস্নেহ

যেথায় মানব, যেথা মানবের গেহ।

বুঝিলাম, ধর্ম দেয় স্নেহ মাতারূপে, পুত্ররূপে স্লেহ লয় পুণঃ, দাতারূপে করে দান, দীনরূপে করে তা গ্রহণ, শিষ্যরূপে করে ভক্তি, গুরু রূপে করে আশীর্বাদ; প্রিয়া হয়ে পাষাণ অন্তরে প্রেম-উৎস লয় টানি, অনুরক্ত হয়ে করে সর্বত্যাগ।",

রবীন্দ্রনাথের ‘চঞালিকা' নাটিকাটি যা পরে নৃত্যনাটিকা হিসেবে সকলের কাছে পরিচিত এটিও বৌদ্ধ সাহিত্য ‘দিব্যাবদান মালা’র অর্ত্তগত ‘শার্দূলর্কণ অবদান’ কাহিনি অবলম্বন করে 
রচিত হয়। মূল কাহিনিটির সারসংক্ষেপ এরকম যা রবীন্দ্রনাথ নিজেও ভূমিকায় তুলে ধরেছিলেন - গল্পের ঘটনাস্থল হল শ্রাবস্তী। প্রভু বুদ্ধ তখন অনাথ পিণ্ডদের উদ্যানে প্রবাস যাপন করছেন। তাঁর প্রিয়শিষ্য আনন্দ একদিন এক গৃহস্থের বাড়িতে আহার করে বিহারে ফিরে এসে তৃষণা অনুভব করছিলেন। দেখলেন এক চন্ডালের কন্যা, নাম প্রকৃতি কুত়ো থেকে জল তুলছে। তার কাছে গিয়ে তিনি জল চাইলে সে জল দিল। আনন্দের রূপ দেখে প্রকৃতি মুগ্ধ হয়ে গেল। তাঁকে পাওয়ার আশা মনে জাগলে উপায়ান্তর না দেখে মায়ের কাছে সাহায্য চেয়েছিলেন। মা যাদুবিদ্যা জানত। তার মা আঙিনায় গোবর লেপে একটি বেদী প্রস্তুত করে সেখানে আগুন জ্বালল এবং মন্ত্রোচ্চারণ করতে করতে একে একে ১০৮ টি অর্কফুল সেই আগুনে আহুতি দিল। আনন্দ সে যাদুর শক্তি রোধ করতে পারলেননা, প্রকৃতি তার বাড়িতে এসে হাজির হল। রাত্রিতে রেদীর উপর আসন গ্রহণ করলে প্রকৃতি তাঁর পুণ্য বিছানা পাততে শুরু করল। আনন্দের মনে তখন পরিতাপ উপস্থিত হুল। পরিত্রানের জন্য ভগবানের কাছে প্রার্থনা জানিয়ে কাঁদতে লাগলেন। ভগবান বুদ্ধ তাঁর অলৌকিক শক্তির মাধ্যনে শিষ্যের অবস্থা জানতে পেরে একটি ব্বীদ্ধমন্ত্র আবৃত্তি করলেন। সেই মন্ত্রের জোড়ে চঞাগিনীর বশীকরণ শক্তি দুর্বল হয়ে পড়ল এবং আনন্দ মঠে ফিরে এলেন। - এই মূল কাহিনিটিকে রবীন্দ্রনাথ একটি বিশেষ সামাজিক কুসংস্কার তথা সংকীর্ণতা থেকে মুক্তি লাভের পথ রচনার কাজে লাগালেন। জাত বিচার অস্পৃশ্যতা এসব সামাজিক আচারের প্রতি রবীন্দ্রনাথথর প্রবল ঘৃণা ছিল। তিনি এই নাটিকাটিতে চঞাল কন্যার হাতে আনন্দকে জলপান করিয়ে সেই সামাজিক ব্যাধি থেকে মুক্তির আলো বিস্তার করলেন। মূল কাহিনিতে প্রকৃতির তীব্র ভোগলালসাও নাটিকাটিতে আনন্দের ক্ষমা সৌন্দর্যে প্রকাশিত হয়েছে। প্রেনের পবিত্র স্পর্শে সমস্ত মলিনতা ধুয়ে মুছে পরিষ্কার হয়ে গেছে। বৌদ্ধধর্মের প্রেম ও করুণার আলো নাটিকাটিতে উদ্ভাসিত হয়ে উঠেছে।

রবীন্দ্রনাথেব কাব্য জগতে অনেক স্থলেই বৌদ্ধ সাহিত্যের পভাব লক্ষ্য করা যায়। সামগ্রিক আলোচনা সম্ভব নয় বলে মাত্র দু-একটি উদাহরণ এখানে তুলে ধরবার চেষ্টা করছি। ‘কথা’ কাব্যের 'পরিশোধ' কবিতাটি এস্থলে উল্লেখযোগ্য। কবিতাটি ‘মহাবস্তু অবদানে’র অন্তর্গত ‘শ্যামাজাতক’ (The Jataka of Sayama) এর কাহিনি অবলম্বনে রচিত। পরবর্তীকালে কবিতাটির নাট্য সংস্করণ ‘শ্যামা’ নৃত্যনাট্য প্রকাশিত হয়। শ্যামা জাতকে ভগবান বুদ্ধ কেন তার বিশ্বস্ত মহিষী যশোধারাকে ত্যাগ করেছিলেন তার কারণ ব্যাখ্যা করতে গিয়ে শ্যামা ও বজ্রসেনের কাহিনির অবতারনা করেছেন। মূল কাহিনিটি সংক্ষেপে এরূপ - প্রাচীনকালে বজ্রসেন নানে তক্ষশীলার এক তরুণ শ্রেষ্ঠা পুত্র অশ্ব বাণিজ্য করতে বারাণসী গিয়েছিল। পতে সে দস্যুদ্বারা আত্রনন্ত হয় এবং তার ঘোড়াগুলি চুরি যায়। তারপর সেই বালক জল নির্গমন পথ দিয়ে কাশী যায় এবং এক জনশূণ্য বাড়িতে শুয়ে থাকে। এদিকে রাজবাড়িতে সেই রাত্রেই চুরি হয়। রাজভৃত্যেরা চোরের খোঁজে সেই শূণ্য গৃহে আসে এবং বজ্রসেনকে চোর সন্দেহ করে তাকে নিয়ে যায়। রাজা তাকে শূলে চড়িয়ে মেরে ফেলবার আদেশ করেন। তাকে নিয়ে যাওয়ার পথে সেই নগরীর ৫্রেষ্ঠা 
গণিকা সুন্দরী শ্যামা তার রূপ লাবত্যে মুগ্ধ হয়ে তার প্রেনে পড়ে যায়। সে যে কোনো মূল্যে বজ্রসেনকে মুক্ত করে আনার জন্য তার দাসীকে পাঠায় এবং প্রচুর অর্থ উপতৌকন দিয়ে দাসী তাকে মুক্ত করে আনে। কিন্তু শুধু অর্থই নয় এই মুক্তির জন্য একটি প্রাণও বলিদান দিতে হয়। এক ধনী টশ্রেষ্ঠির একমাত্র পুত্র বারো বছরের জন্য শ্যামাকে ত্রয় করে তার সঙ্গে বাস করছিল। বারো বছরুরে মট্যে দশ বছর কেটে গেছে। আর দুবছর বাকী আছে। শ্যামা কৌশলে বজ্রসেনের পরিবর্তে ঐ নিরীহ ছেলেটিকে পাঠিয়ে দেয় এবং ঘাতকেরা তাকে বধ করে। শ্যামা বজ্রসেনকে প্রেনে আপ্লুত করে রাখার চেষ্টা করে। বজ্রসেন শ্যামার নির্মমতার কথা জানতে পারে এবং তার মনে ভীতির সঞ্চার হয়। তার ধারণা হয়, শ্যামা বোধহয় কিছুদিন বাদে তাকেও নেরে ফেলবে। সে কৌশলে শ্যামার হাত থেকে মুক্তি পাবার চেষ্টা করে। একদিন জলক্রীড়ার অবসরে সে শ্যামাকে মদ্যপান করিয়ে অচেতন অবস্থায় ফেলে রেখে পালিয়ে যায়। পরে শ্যামার দাসীরা শ্যামাকে রেই অবস্থায় ঘরে নিয়ে আলে, এবং পরিচর্যা করে সুস্থ করে তোলে। সুস্থ হয়েই শ্যামা অতি দ্রু০ত চঞালদের সাহায্যে এক সদ্যমৃত নরদেহ যোগাড় করে এবং সেই মৃতদেহ ধরে ভান করে কান্নাকাটি করতে থাকে। সকলে জানতে পারল যে শ্যামার প্রেমীক সেই শ্রেষ্ঠিপুত্র মারা গেছে। শ্যামা বজ্রসেনের প্রেন্মে উন্মাত্ত হয়ে এসব ঘটনা সংঘটিত করে তার আগমন প্রতীক্ষায় উদাসভাবে বসে থাকে। শ্যামার আলুথালু অবস্থা দেখে সবাই মনে করে সে বোধহয় ঐ শ্রেষ্ঠি পুত্রের জন্য উন্মাদ হয়ে গেছে। এরপর তক্ষশীলা থেকে বারাণসীতে আসা একদল নটের কাছে শ্যামা বজ্রসেনের প্রতি স্মৃতি বিজড়িত একটি শ্লোক লিখে পাঠায়, কিন্তু বজ্রসেন প্রত্যুত্তরে শ্যামাকে কোনোরূপ প্রশ্রয় দেয় না এবং তার সংস্পর্শ এড়ানোর জন্য দূরদেশে চলে যাওয়ার সিদ্ধান্ত নেয়। - এই ‘মহাবস্তু অবদান’ এর ‘শ্যামা জাতক’ থেকে উদ্বুদ্ধ হয়ে রবীন্দ্রনাথ সৃষ্টি করেছিলেন তাঁর ‘পরিশোধ’ কবিতা বা ‘শ্যামা’ নৃত্যনটট্য। রবীন্দ্রনাথ মূল কাহিনি থেকেও আকর্ষণীয় করে তুলেছেন এই শ্যামা নৃত্য নাটকটিকে, কিন্তু সর্ব্বাপরি মূল কাহিনীর ছায়া এবং বৌদ্ধধর্মের মানবিক আবেদনে ঋদ্ধ হয়ে উঠেছে রবীন্দসৃষ্টি তা অনস্বীকার্য।

‘কথা’ কাব্যের ‘পূজারিনী’ কবিতাটির মূল কাহিনিও ‘অবদান শতক’ থেকে নেওয়া।' রাজেন্দ্রলাল মিত্র অনূদিত কাহিনিটি সংক্ষেপে এরূপ - রাজা বিম্বিসার ভগবান তথাগতের কাছে সত্য সম্বন্ধে জ্ঞানলাভ করেন এবং তাঁর কেশ ও নটের উপর স্তূপ নির্মাণ করে রাজ অন্তঃপুরে প্রতিষ্ঠিত করেন। রাজপরিচারিকারা প্রত্যহ ,েই স্তূপ মার্জন করতো। অজাতশত্রঃ পিতার বর্তমানেই রাজা হন এবং ঐ স্টূপ বেদীর মার্জনা বা কোনো প্রকারে বুদ্ধের পজার আয়োজন করলে মৃত্যুদণ বিধান করেন। শ্রীমতী নামে এক দাসী জীবনের মায়া তুচছ করে বুদ্ধের স্তূপ মার্জনা করেন এবং একসার দীপমালায় সেই স্থান আলোকিত করেন। এতে ত্রেনধান্ধ হয়ে অজাতশত্রং তাঁকে বধ্যভূমিতে নিয়ে যেতে আদেশ করেন। মৃত্যুর পর তিনি দেবকন্যা হয়ে বেণুবনে ভগবান বুদ্ধের সম্মুখে অবতীর্ণা হন এবং মানুযের দুঃখের পর্বতকে জ্ঞন বজ্রের দ্বারা বিদীর্ণ করে কাম্যবস্তু সকল লাভ করেন।’’ - ‘অবদান শতকে’র এই 
কাহিনি অবলম্বন কট়র রবীল্দ্রনাথ তাঁর অনবদ্য কবিতা ‘পূজারিনী’ রচনা করেন। যদিও ভাব, ভাযা ও ছন্দ প্রয়োদের চাতুর্যে কবিতাটি ন্যেলিক আবেদনের দাবি রাখে, কিন্তু কবিতাটির অনেকাংশই মূলের হুবহ অনুকরণ বলা যেতে পারে। যেমন শ্রীমতীর শেয পরিণতিটি-

"সেদিন শুভ্র পাযাণ ফলকে

পড়িল রক্তলিখা।

সেদিন শারদ স্বচ্ছ নিশীথথ

প্রসাদ কাননে নীরবে নিভৃতে

স্টূপ পদমূূলে নিবিল চকিতে

শেষ আরতির শিখা।"’২

মূল কাহিনির বিশ্বস্ত অনুসরণ আরও দেখা যেতে পারে, যেমন কবিতাটির প্রথম স্তবক :

$$
\begin{aligned}
& \text { “নৃপতি বিম্বিসার } \\
& \text { নমিয়া বুদ্ধে মাগিয়া লইল } \\
& \text { পদনখকণা তাঁর। } \\
& \text { স্থাপিয়া নিভৃত প্রাসাদ কাননে } \\
& \text { তাহারি উপরে রহিলা যতনে } \\
& \text { অতি অপরূপ শিলাময় স্তুপ। } \\
& \text { শিল্পশোভায় সার।",* }
\end{aligned}
$$

এরকম আরও অনেক উদাহরণ দেওয়া যেনে পারে, কিন্তু বাহুন্যের জন্য অন্য উদাহরণ বর্জন করা গেল।

রবীল্দ্রনাটের একই কাব্যের ‘শ্রেষ্ঠ ভিক্ষন’ কবিতাটির শিরোনানে ‘অবদানশতক’ এবং বুদ্ধশিষ্য 'অনাথপিকদ্' এর উল্লেখ আছে। মূল 'অবদান শতকে'র পঞ্চপঞ্চাশৎ অবদানে ('বস্ত্রম্') ভিক্ষু অনাথ পিগুদের ভিক্ষা সংগ্রহের কাহিনি আছে। রাজেন্দ্রলাল মিত্রের অনুবাদ্দ কাহিনিটি যেভাবে আছে তা এরকম-

"Anathpindala obtained permission from the king to solicit alms for the Lord, for the benefit of the whole population of the city. Oh an elephant rode the patriarch receiving metallic vessels, bracelets and other ornaments as alms for his neighbours. A poor woman, who has an only cloth, threw it over the elephant from behind a hedge. The beggar knew instantly what the matter was, and bestowed on her rich presents. She went to the Lord and

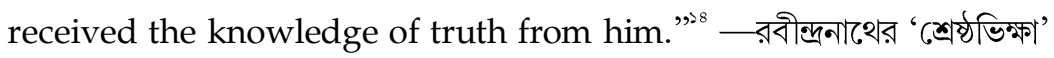
কবিতায মূল কাহিনি অক্ষুক্ন আছে, যদিও কঙ্গনা ও প্রকাশ কৌশশলের দিক্ থেকে এটি একটি নতুন সৃষ্টি বনে মনে হয়। মূনের ভিক্ষু অনাথাপিঞ্ডদ রাজ অনুনোদন নিয়ে 
সর্বমানবের কল্যাণের জন্য ভিক্ষায় বেরিয়েছিলেন। নানা ধনরত্নে তাঁর ভিক্ষার ঝুলি ভরে উঠেছিল; কিন্তু রবীন্দ্রনাথের অনাথপিঞ্ডদ এই ধনরত্ন ইত্যাদিতে তৃপু হতে পারেন নি, তিনি চেয়েছেন নিঃস্বার্থ হৃদয়ের দান, যা ভিক্ষুশ্রেষ্ঠ ভগবান যুদ্ধের যোগ্য দান। তাই তিনি নগরী ছেড়ে গেছেন অদূরবর্তী একটি কাননে যেখানে তাঁর মনের মতো দান পেয়েছেন তিনি। এক দরিদ্র রমনী তার একমাত্র পরিধেয় বস্ত্র সে দান করেছে-

“অরণ্য আড়ালে রহি কোনো মতে

একমাত্র বাস নিল গাত্র হতে,

বাহটি বাড়াড়ে ফেলি দিল পথে ভূতলে। ৷»

‘অবদান শতরে’র কাহিনির মূলভাবের সঙ্গে সাযুজ্য রেখে কবিতাটি রচনা করেছেন কবি। বৌদ্ধ সাহিত্যের এই প্রভাব তাঁর আরও অনেক কবিতায় (অভিসার, মস্তকবিত্রঃয়, মূল্যপ্রাপ্তি, সামান্য ক্ষতি) রয়েছে। এই কবিতাগুলোর আলোচনা প্রবন্ধের বিস্তৃতির ভয়ে করা গেলনা।

সুতরাং মূল কথা হল বৌদ্ধ দর্শন ও ব্বেদদ্ধ সাহিত্যে রবীন্দ্রনাথের অশেশ আগ্রহ ছিল এবং তারই ফলশ্রুতিস্বরূপ তাঁর বিভিন্ন রচনায় বৌদ্ধ দর্শন ও সাহিত্যের প্রভাব লক্ষ্য করা যায়। বুদ্ধের জীবন ও বাণী কবিকে গভীরভাবে স্পর্শ করেছিল। বৌদ্ধ দর্শনের জীবনের যে দুঃখবাদ তা রবীন্দ্র সৃষ্টিতে না থাকলেও বুদ্ধের নৈত্রী, প্রেম ও করুণায় তিনি অভিভূত হয়েছিলেন এবং তাঁর যে সাহিত্যেই বৌদ্ধ প্রভাব আমরা দেখতে পাই সেখানেই মানবিকতার জয় ঘোষণা হয়েছে, প্রেনের অমৃত্পর্র্রে সব অমৃতময় হয়ে উঠেছে।

ড্’ পম্পা মজুমদার তাঁর ‘রবীন্দ্র সংস্কৃতির ভারতীয় রূপ ও উৎস’ গ্রন্থে রবীন্দ্রনাথের বৌদ্ধ সাহিত্যের প্রতি যে গভীর শ্রদ্ধাবোধ ছিল তা উল্লেখ করতে গিয়ে লিখেছেন-“.....বৌদ্ধ শাস্ত্র আলোচনার জন্য তিনি বিশ্বভারতীতে ভিক্ষুদের আমন্ত্রণ ও তাঁদের বক্তৃতার আয়োজন করে সোৎসাহে সেগুলিতে উপস্থিত থাকতেন। উপর বুদ্ধের গভীর প্রভাব সম্পর্কে আলোচনা করতে গিয়ে বলেছেন- "রবীন্দ্রনাথ বৌদ্ধদর্শনের যে তত্ত্বতীর্থে পরিভ্রমণ করিয়াছেন তাহাকে নিজের জীবনে গ্রহণ করিয়াছেন।"’a তাত্ত্বিকদের এসকল উক্তির মাধ্যমে আমরা সহজেই অনুমান করতে পারি যে রবীন্দ্রনাথ কীভাবে বৌদ্ধ দর্শন ও সাহিত্যের দ্বারা প্রভাবান্বিত হয়েছিলেন।

\section{উন্লেখপঞ্জি:}

১) ঠাকুর রবীন্দ্রনাথ - বুদ্ধদেব, পৃ: ৬

২) ঘটক কল্যাণীশঙ্কর - রবীন্দ্রনাথ ও সংস্কৃত সাহিত্য, বর্ধমান বিশ্ববিদ্যালয়, ২০০০, পৃ: ৩১৮ 
৩) Ed. by Mitra Rajendralal - The Sanskrit Buddhist literature of Nepal, Calcutta-1882 - 'The Story of Kusa', p. 142

৪) ঘটক কল্যাণীশঙ্কর - ্রাগক্ত, পৃ: ৩২০

৫) তদেব - পৃ: ২৪

৬) তদেব - পৃ: ৩০

৭) ঠাকুর রবীন্দ্রনাথ - রবীন্দ্ররচনাবলী, বিশ্বভারতী, ২০০৩, মালিনী, পৃ: ৩৪১

৮) Ed. by Mitra Rajendralal - প্রাগুক্ত, p. 223

৯) ঘটক কল্যাণীশঙ্কর _ _ প্রাগুক্ত, পৃ: ৩০৬

১০) তদেব - পৃ:৩০৪

১১) তদেব - পৃ:৩০৪

১২) ঠাকুর রবীন্দ্রনাথ ্রাগুক্ত - ‘কথা' - পূজারিনী, পৃঃ ৩১

১৩) তদেব - পৃ:২৯

১8) Ed. by Mitra Rajendralal - প্রাগুক্ত, p. 33

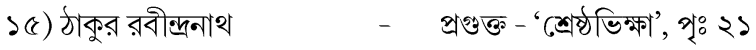

১৬) মজুমদার ড’ পম্পা - রবীন্দ্র সংস্কৃতির ভারতীয় রূপ ও উৎস, দে’জ পাবলিশিং, কলকাতা, পৃ: ৪৭১

১৭) দাশ ডঃ আশা - বৌদ্ধধর্ম ও রবীন্দ্রনাথ, কঙ্লোল প্রকাশনী, কলকাতা, পৃঃ ২ 